\title{
Projetando Abordagens Analíticas Imersivas para a Exploração de Dados Espaço-Temporais
}

\author{
Jorge Wagner, Luciana Nedel \\ Universidade Federal do Rio Grande do Sul \\ \{jawfilho, nedel\}@inf.ufrgs.br
}

\begin{abstract}
Resumo: Um dos maiores desafios na computação atualmente é extrair informações relevantes de conjuntos de dados cada vez maiores. Técnicas de visualização de dados permitem aplicar as habilidades humanas de compreensão visual e conhecimento do domínio a este processo. A hipótese deste trabalho é que ambientes imersivos e estereoscópicos de Realidade Virtual (RV), combinados com interação natural, suportarão a exploração de representações de dados espaço-temporais inerentemente tridimensionais melhor do que ambientes desktop convencionais. Investigando-se esta hipótese, pretende-se identificar as escolhas de projeto mais eficientes para este tipo de aplicação em termos de interação e colaboração, através de sucessivas avaliações controladas com usuários empregando conjuntos de dados reais. Neste artigo, discute-se como resultados iniciais confirmam o potencial deste tipo de abordagem e quais são os próximos passos nesta pesquisa.
\end{abstract}

Palavras-chave: Visualização imersiva; Cubo espaço-temporal; Visualização de trajetórias.

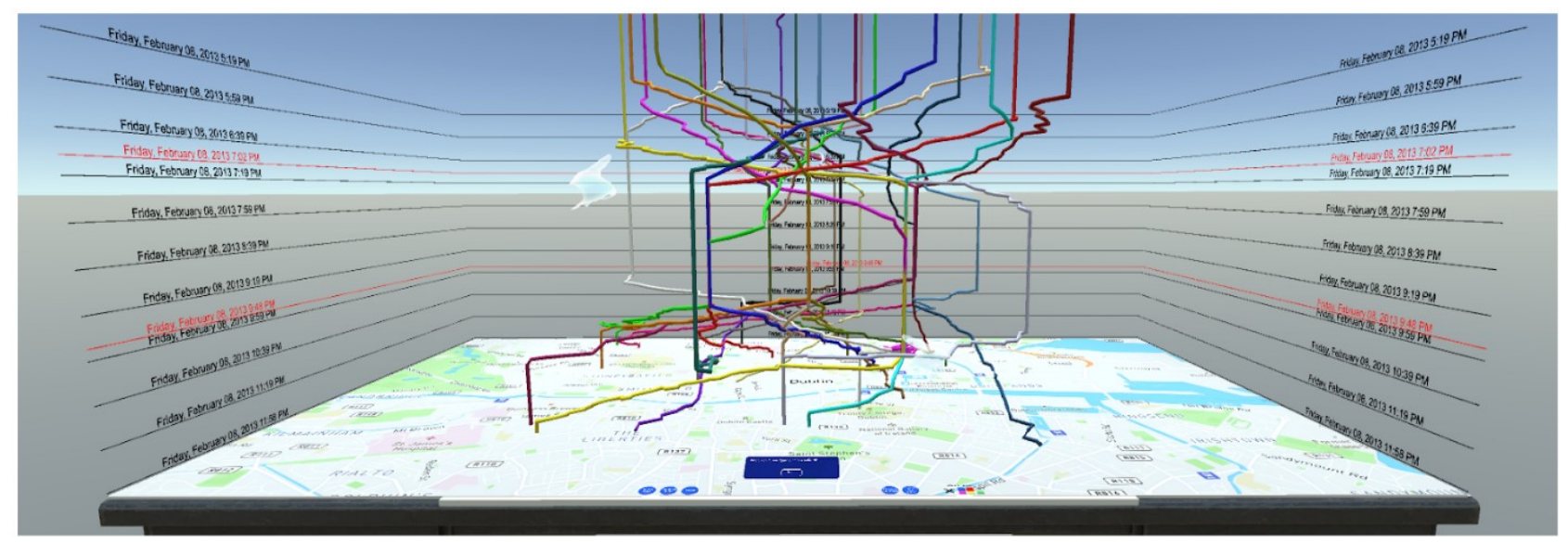

Figura 1. Na primeira abordagem imersiva proposta neste trabalho para explorar dados espaço-temporais, trajetórias de movimentação dispostas tridimensionalmente ao longo do tempo e do espaço foram posicionadas sobre uma mesa virtual, a qual também oferece controles tangíveis.

\section{Introdução}

Entender como as posições de objetos ou pessoas variam ao longo do tempo e extrair padrões significativos e conclusões a partir destes dados é um tópico de interesse crescente para diversas categorias de usuários, desde pessoas comuns planejando seus deslocamentos até pesquisadores de geografia humana e agentes tomadores de decisões em órgãos públicos interessados em se preparar para possíveis situações futuras ou em analisar as movimentações passadas. Atualmente, dispositivos móveis equipados com GPS, redes de telecomunicação [1] e até mesmo redes sociais [2] são capazes de facilmente coletar grandes e detalhados conjuntos de dados registrando a movimentação de seus usuários ao longo do tempo. Aplicações como cidades inteligentes, planejamento de transportes, estudos comportamentais, controle de epidemias, bem como aquelas que visam aumentar o engajamento dos cidadãos na governança pública podem se beneficiar fortemente da análise de grandes volumes de dados como estes. A alta complexidade e heterogeneidade destes dados invariavelmente requerem a integração da percepção e conhecimento de domínio humano às técnicas automatizadas de análise de dados. Todavia, visualizar atributos e padrões espaço-temporais essenciais nestes conjuntos de dados segue um desafio.

Representações baseadas em mapas bidimensionais focam na natureza espacial dos dados e, mesmo com a ajuda de animações, tornam difícil a observação de características como durações e velocidades variáveis de movimento, locais e durações de paradas e locais de encontro entre diferentes indivíduos (quando estes compartilham a mesma posição no tempo e no espaço). Representações tridimensionais são uma alternativa para abordar de forma mais adequada a natureza temporal destes dados, por exemplo ao empregar o eixo perpendicular ao mapa para representar a componente do tempo, o que resulta em um Cubo Espaço-Temporal (STC - Space-Time Cube).

No entanto, da mesma forma que outras representações tridimensionais de dados, o STC possui limitações bem conhecidas em termos de percepção e interação quando usado em ambientes convencionais do 
tipo desktop. Estas limitações são resultado das dificuldades em estimar distâncias e profundidades a partir de apenas referências visuais monoculares, e da incompatibilidade em termos de controle entre um ambiente $3 \mathrm{D}$ e dispositivos de interação $2 \mathrm{D}$ como o mouse, além dos desafios introduzidos pela oclusão e poluição visual. Isto se torna ainda pior quando se leva em conta que especialistas no domínio dos dados tipicamente não são - e não deveriam precisar ser treinados em manipulações 3D. Trabalhos anteriores apontaram que especialistas haviam reclamado especificamente da longa curva de aprendizado para a utilização do STC [3].

\section{Trabalhos Relacionados}

Originalmente proposto por Hägerstrand [4], o STC foi revisitado por Kraak [5] no contexto de um ambiente interativo de geovisualização e, posteriormente, aplicado a diversos domínios, como dados de eventos [6], trajetórias de navios [7], análise de tráfego aéreo [8] e dados de posicionamento de telefones móveis [3].

Alguns esforços iniciais para implementar representações imersivas de dados de movimento, como o STC, foram relatados por Theuns [9] usando um protótipo baseado em capacete de Realidade Virtual (RV), e por Saenz et al. [10] usando um capacete de Realidade Aumentada (RA). Moran et al. [11] também exploraram uma abordagem em RV para a visualização de posts geo-posicionados do Twitter originados no campus do MIT. Os tweets foram distribuídos em uma reprodução virtual do campus e a representação visual específica de cada um foi determinada de acordo com o seu conteúdo. Na aplicação HoloMaps, tweets geoposicionados e informações de tráfego foram apresentados em tempo real um modelo de cidade 3D, usando o capacete de RA HoloLens [12]. Por fim, no sistema de RA GeoGate, Ssin et al. [13] combinaram uma tela 2D do tipo tabletop com "hologramas" tridimensionais em RA para visualizar trajetórias no domínio marítimo. Em razão do campo de visão limitado dos dispositivos de RA atuais, o STC foi exibido em tamanho pequeno e a sua posição controlada através da movimentação de um dispositivo tangível, o qual funcionou como um filtro espacial. O GeoGate foi capaz de reduzir erros em tarefas onde os usuários tinham que correlacionar diferentes fontes de dados.

\section{Metodologia}

O desafio de pesquisa que se propõe para este projeto consiste no planejamento e avaliação de representações imersivas eficientes para dados espaço-temporais. A hipótese é que ambientes de visualização imersiva [14] baseados em capacetes de RV e combinados com interação 3D natural melhor suportarão a exploração visual de tais dados tridimensionais altamente complexos, reduzindo a curva de aprendizado e as dificuldades de interação, como as relatadas anteriormente por Kveladze et al. [3].

A abordagem inicial é baseada na melhoria e expansão da técnica VirtualDesk, proposta em estudos anteriores voltados para a visualização imersiva de dados multidimensionais [15], e na sua combinação com metáforas complementares tais como voo virtual e caminhamento real para exploração egocêntrica dos dados. Na VirtualDesk, os dados são exibidos em pequena escala sobre uma reprodução virtual da mesa real de trabalho do analista, possibilitando interações incorporadas e tangíveis e oferecendo referências mais fortes de estereopsia e propriocepção. Anteriormente, esta abordagem resultou em benefícios de precisão em relação a uma alternativa desktop para tarefas de percepção de distâncias e densidades, ao mesmo tempo em que adicionou pouca ou nenhuma demanda temporal e não causou nem desconforto nem enjoo, até então uma preocupação séria em aplicações imersivas. Tendo em vista a natureza heterogênea dos dados espaçotemporais, múltiplas visualizações coordenadas serão necessárias, podendo ser posicionadas na superfície ou em torno da mesa virtual do analista.

Esta pesquisa segue uma estratégia iterativa de projeto e avaliação, utilizando avaliações controladas com usuários, tanto com participantes especialistas no domínio dos dados quanto com leigos.

\section{Resultados Iniciais}

A primeira etapa da pesquisa consistiu em validar o potencial de ambientes imersivos. Para isso, implementou-se um primeiro protótipo (ver Figuras 1, 2 e 3) e conduziu-se uma avaliação com usuários.

Em um experimento controlado [16], 20 participantes completaram 7 tarefas de diferentes níveis de dificuldade tanto no protótipo imersivo quanto em um ambiente desktop convencional. O primeiro atingiu uma pontuação de usabilidade significativamente mais elevada no questionário SUS [17] (82,3 vs. 62,1) e conquistou a preferência da maioria dos participantes 19 o consideraram mais engajador, 18 mais intuitivo e 13 mais rápido. Além disso, a incidência de desconforto foi muito baixa (incremento médio de 2,8 pontos no questionário SSQ após o experimento), e a carga mental medida pelo questionário NASA-TLX significativamente reduzida (de 41,6 para 32,4).

Por fim, também foram coletadas recomendações de melhorias e novas funcionalidades a partir de uma colaboração em andamento com pesquisadores da geografia [18]. Estas recomendações incluem novas ferramentas, como planos de corte e filtros mais avançados, bem como o enriquecimento dos dados com uma maior variedade de atributos semânticos.

\section{Discussão}

As próximas etapas desta pesquisa incluem avaliações mais detalhadas com especialistas no domínio dos dados, e a aplicação da abordagem a conjuntos de dados espaço-temporais reais em diferentes domínios, tais como dados de mobilidade urbana e de saúde pública. Diferentes categorias de dados (e.g., dados de eventos, dados do tipo origem-destino, ou trajetórias de GPS) e maiores volumes de dados resultarão em diferentes requisitos de projeto, que precisarão ser suportados. 

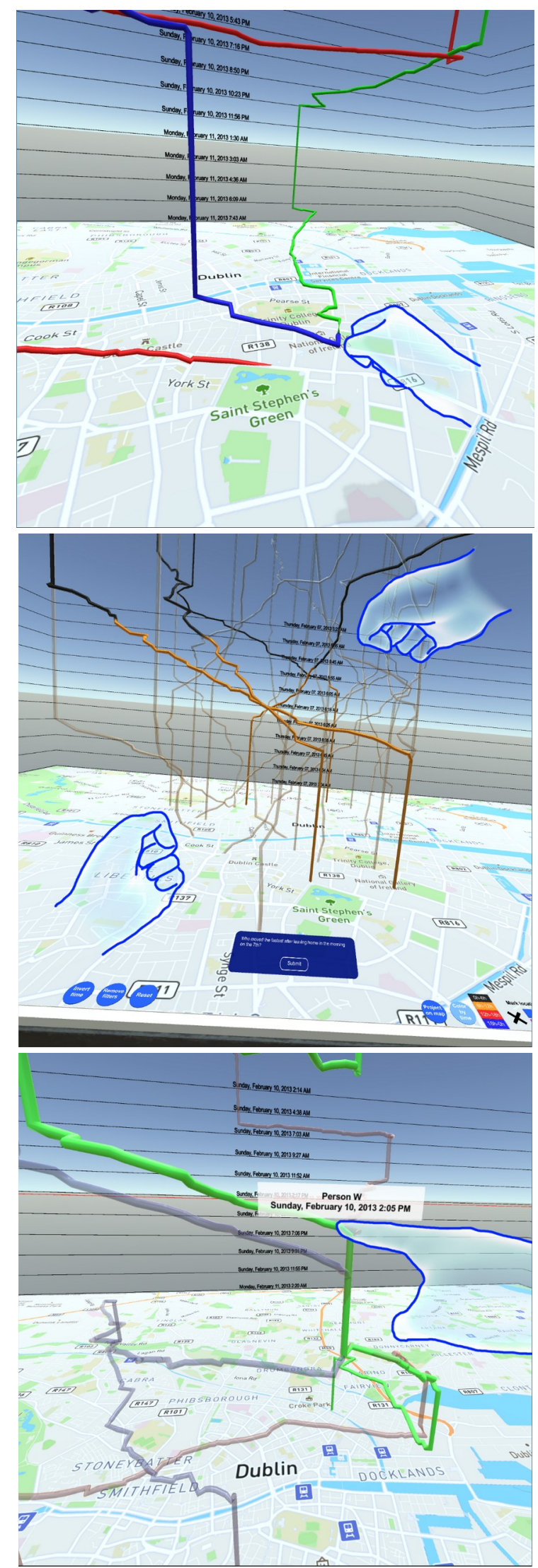

Figura 2. No cubo espaço-temporal imersivo, todas ações são implementadas por meio de gestos intuitivos, como segurar (topo), esticar (centro) e encostar (baixo). Contornos das mãos foram adicionados à figura para maior clareza

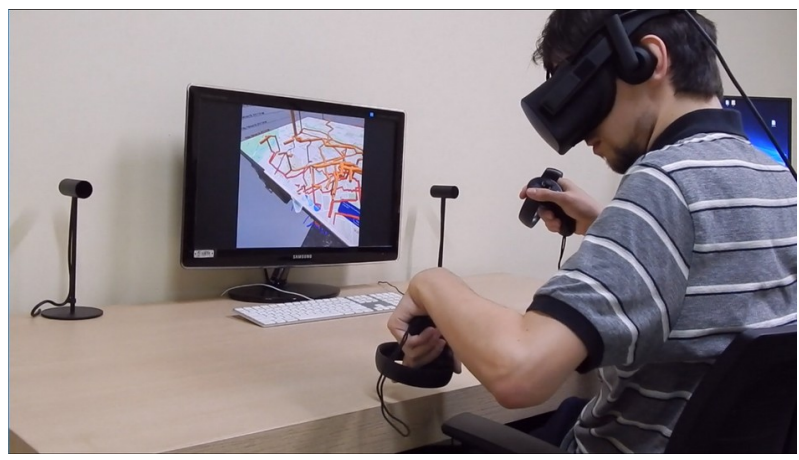

Figura 3. Ao reproduzir a mesa real do analista de dados no ambiente virtual, permite-se a interação tangível com comandos dispostos na superfície da mesma, além de agregar uma referência do mundo real.

Levando em conta o processo de trabalho típico de analistas de dados, também serão investigadas diferentes possibilidades de técnicas de interação e, especialmente, colaboração (tanto local quanto remota) e seus efeitos no desempenho analítico geral.

Espera-se que esta pesquisa culmine em um ambiente analítico imersivo completo, que suporte metáforas de exploração complementares e que seja capaz de auxiliar diferentes tipos de usuários, incluindo autoridades municipais, planejadores urbanos e pesquisadores em seus processos de tomada de decisões. Baseando-se no uso de dispositivos de baixo custo, este ambiente também deve ser capaz de engajar usuários regulares na exploração casual de dados [19].

De uma forma mais ampla, essas avaliações também resultarão em novas diretrizes para a construção de aplicações eficientes para visualização imersiva de dados [14], e para o projeto de técnicas apropriadas de interação e colaboração neste contexto.

\section{Conclusão}

Ambientes imersivos de Realidade Virtual mostram-se úteis para atividades de análise de grandes volumes de dados, possibilitando que analistas interajam de forma natural com dados tridimensionais. Resultados iniciais confirmaram que um Cubo Espaço-Temporal imersivo pode proporcionar maior usabilidade e menor carga mental em comparação a um ambiente desktop convencional. A partir de avaliações mais detalhadas com especialistas e novos conjuntos de dados, espera-se definir as abordagens mais adequadas para a visualização e interação com os dados nestes ambientes, de forma que possam auxiliar analistas e tomadores de decisão em ambientes reais.

\section{Agradecimentos}

Agradecemos financiamento recebido do Conselho Nacional de Desenvolvimento Científico e Tecnológico (CNPq) e de Global Affairs Canada. O presente trabalho foi realizado com apoio da Coordenação de Aperfeiçoamento de Pessoal de Nível Superior - Brasil (CAPES) - Código de Financiamento 001. 


\section{Bibliografia}

[1] Calabrese, F.; Ferrari, L.; Blondel, V. D. (2015) Urban sensing using mobile phone network data: A survey of research. ACM Computing Surveys 47(3):1-20. DOI: $10.1145 / 2655691$.

[2] Noulas, A.; Scellato, S.; Mascolo, C.; Pontil, M. (2011) An empirical study of geographic user activity patterns in foursquare. Proc. Fifth International AAAI Conference on Weblogs and Social Media.

[3] Kveladze, I.; Kraak, M.-J.; Van Elzakker, C. P. (2015) The space-time cube as part of a geovisual analytics environment to support the understanding of movement data. International Journal of Geographical Information Science 29(11):2001-2016. DOI: 10.1080/13658816.2015.1058386.

[4] Hagerstraand, T. (1970) What about people in regional science? Papers in Regional Science 24(1):7-24. DOI: 10.1007/BF01936872.

[5] Kraak, M.-J. (2003) The space-time cube revisited from a geovisualization perspective. Proc. 21st International Cartographic Conference. Citeseer, pp. 1988-1996.

[6] Gatalsky, P.; Andrienko, N.; Andrienko, G. (2004) Interactive analysis of event data using space-time cube. In: Proc. International Conference on Information Visualisation (IV). IEEE, pp. 145-152 .

[7] Andrienko, N.; Andrienko, G. (2013) Visual analytics of movement: An overview of methods, tools and procedures. Information Visualization, 12(1):3-24. DOI: $10.1177 / 1473871612457601$.

[8] Buschmann, S.; Trapp, M.; Dollner, J. (2016) Animated visualization of spatial-temporal trajectory data for airtraffic analysis. The Visual Computer 32(3):371-381. DOI: 10.1007/s00371-015-1185-9.

[9] Theuns, J. (2017) Visualising origin-destination data with virtual reality: Functional prototypes and a framework for continued VR research at the itc faculty. B.S. Thesis, University of Twente.

[10] Saenz, M.; Baigelenov, A.; Hung, Y.-H.; Parsons, P. (2017) Reexamining the cognitive utility of 3D visualizations using augmented reality holograms. Proc. IEEE VIS Workshop on Immersive Analytics. IEEE

[11] Moran, A.; Gadepally, V.; Hubbell, M.; Kepner, J. (2015) Improving big data visual analytics with interactive virtual reality. Proc. IEEE High Performance Extreme Computing Conference. p. 1-6. DOI: 10.1109/HPEC.2015.7322473.

[12] Hills-Duty, R. (2017) Taqtile are creating new holomaps for the hololens. Online: https://www.vrfocus.com/2017/07/taqtile-are-creatingnew-holomapsfor-the-hololens. Acesso em 22/10/17.

[13] Ssin, S. Y.; Walsh, J. A.; Smith, R. T.; Cunningham, A.; Thomas, B. H. (2019) Geogate: Correlating geo-temporal datasets using an augmented reality space-time cube and tangible interactions. Proc. 26th IEEE Conference on Virtual Reality and 3D User Interfaces. p. 210-219. DOI: 10.1109/VR.2019.8797812.

[14] Chandler, T.; Cordeil, M.; Czauderna, T.; Dwyer, T.; Glowacki, J.; Goncu, C.; Klapperstueck, M.; Klein, K.; Marriott, K.; Schreiber F. et al. (2015) Immersive analytics. Proc. 2015 Big Data Visual Analytics (BDVA). p. 1-8. DOI:10.1109/BDVA.2015.7314296.

[15] Wagner Filho, J. A.; Freitas, C. M.; Nedel, L. (2018) VirtualDesk: A Comfortable and Efficient Immersive Information Visualization Approach. Computer Graphics Forum 37(3):415-426. DOI: 10.1111/cgf.13430.

[16] Wagner Filho, J. A.; Stuerzlinger, W.; Nedel, L. (2019) Evaluating an immersive space-time cube geovisualization for intuitive trajectory data exploration. IEEE Transactions on Visualization and Computer Graphics 26(1): 514-524. DOI: 10.1109/TVCG.2019.2934415.

[17] Brooke, J. (1996) SUS-A quick and dirty usability scale. Usability Evaluation in Industry, ch. 21.

[18] Wagner Filho, J. A.; Freitas, C. M. D. S.; Nedel, L. (2019) Comfortable immersive analytics with the virtualdesk metaphor. IEEE Computer Graphics and Applications 39(3): 41-53. DOI: 10.1109/MCG.2019.2898856.

[19] Pousman, Z.; Stasko, J.T (2007) Casual information visualization: depictions of data in everyday life. IEEE Transactions on Visualization and Computer Graphics, 13(6):1145-1152. DOI: 10.1109/TVCG.2007.70541. 